\title{
Breast tumor aromatase: functional role and transcriptional regulation
}

\author{
S Chen, D Zhou, T Okubo, Y-C Kao and C Yang \\ Division of Immunology, Beckman Research Institute of the City of Hope, Duarte, California 91010, USA \\ (Requests for offprints should be addressed to $\mathrm{S}$ Chen)
}

\begin{abstract}
Aromatase has been shown to be expressed at a higher level in human breast cancer tissue than in normal breast tissue, by means of enzyme activity measurement, immunocytochemistry, and RT-PCR analysis. Cell culture including MCF-7 breast cancer cells, animal experiments using aromatasetransfected breast cancer cells, and transgenic mouse studies have demonstrated that estrogen production in situ plays a more important role than circulating estrogens in breast tumor promotion. In addition, tumor aromatase is believed to be able to stimulate breast cancer growth through both autocrine and paracrine pathways, as demonstrated by a three-dimensional cell culture study. RT-PCR and gene transcriptional studies have revealed that the aromatase promoter is switched from a glucocorticoid-stimulated promoter, I.4, in normal tissue to cAMP-stimulated promoters, I.3 and II, in cancerous tissue. Recently, we identified and characterized a cAMP-responsive element (CREaro) upstream from promoter I.3 by DNA deletion and mutational analyses. Our results from promoter functional analysis also demonstrated an interaction between the CREaro and the silencer element (S1) that was identified previously in our laboratory. In the presence of cAMP, the positive regulatory CREaro can overcome the action of the silencer on the function of promoter I.3. On the basis of results generated from our own and other laboratories, we propose that, in normal breast adipose stromal cells and fibroblasts, aromatase expression is driven by promoter I.4 (glucocorticoid dependent), and that the action of promoters I.3 and II is suppressed by the silencer negative regulatory element. However, in cancer cells and surrounding adipose stromal cells, the cAMP level increases, and aromatase promoters are switched to CAMP-dependent promoters - I.3 and II. Furthermore, we applied the yeast one-hybrid screening method to search for proteins interacting with the silencer element, S1. The major protein identified was ERR $\alpha-1$; however, SF-1, which is present in the ovary, is not detected in breast cancer tissue. Using a reporter plasmid with the aromatase genomic fragment containing promoter I.3 and S1, in breast cancer SK-BR-3 cells, ERR $\alpha-1$ was found to have a positive regulatory function. It is believed that the silencer element in the human aromatase gene may function differently in different tissues, as a result of distinct expression patterns of transcription factors.
\end{abstract}

Endocrine-Related Cancer (1999) 6 149-156

\section{Aromatase expression in breast cancer tissues}

James et al. (1987) reported that aromatase activity, when measured in vitro, was found to be higher in breast tumors than in the fat next to the tumor or in normal breast fat. In addition, Miller \& O’Neill (1987) found a highly significant correlation between aromatase activity and the presence of tumors in individual quadrants of breast tissue. Miller et al. (1997) further demonstrated that fibroblast cultures derived from tumor-bearing quadrants tended to display higher aromatase activity than cultures derived from non-tumor-bearing quadrants. By quantitative PCR analysis, adipose stromal cells surrounding the cancer cells have also been shown to contain aromatase mRNA at a higher level than that in adipose stromal cells in noncancerous areas (Bulum et al. 1993, Harada 1997). The aromatase mRNA levels $\left(4.53 \pm 0.66 \times 10^{-3} \mathrm{amol} / \mu \mathrm{g} \mathrm{RNA}\right)$ in the breast cancer tissue were found to increase significantly compared with non-malignant breast tissues $\left(1.73 \pm 0.40 \times 10^{-3} \mathrm{amol} / \mu \mathrm{g}\right.$ RNA; Harada 1997). It has also been reported that the concentrations of estrogens in breast tumor tissues were found to be several-fold greater than those in plasma in postmenopausal patients (Pasqualini et al. 1996). These results support tumor accumulation and in situ synthesis of estrogens. We have 
detected aromatase mRNA in 67 of 70 breast tumor specimens (Zhou et al. 1996b).

Immunocytochemical analysis from our laboratory first identified the presence of aromatase in breast cancer epithelial and stromal cells (Esteban et al. 1992), while others had reported the presence of aromatase only in the stromal tissue (Santen et al. 1994, Sasano et al. 1994). Our findings have been confirmed by independent in situ hybridization studies and cell proliferation assays showing that aromatase is expressed in breast cancer epithelial cells (Lu et al. 1996).

Therefore, it can be stated that aromatase is expressed in breast cancer tissue, probably at a higher level than in normal breast tissue, as demonstrated by enzyme activity measurement, immunocytochemistry, and RT-PCR analysis.

\section{Consequence of aromatase expression in breast tumors}

The MCF-7 breast cancer cell line is estrogen receptor (ER) positive and is often used as a model cell line to demonstrate the estrogen dependence of breast cancer growth. Although the aromatase gene was found to be amplified in MCF-7 cells, as shown by Southern and DNA slot blot analyses (Zhou et al. 1993), our laboratory has reported that aromatase activity in MCF-7 cells (specific activity, $0.08 \mathrm{pmol}\left[{ }^{3} \mathrm{H}\right] \mathrm{H}_{2} \mathrm{O}$ formed/h per mg) is at a level similar to that in skin fibroblasts and is significantly lower than that in human placenta choriocarcinoma JAR cells (i.e., $29 \mathrm{pmol}\left[{ }^{3} \mathrm{H}\right] \mathrm{H}_{2} \mathrm{O}$ formed/h per $\mathrm{mg}$ ). Kitawaki et al. (1992) reported that, by converting androgen to estrogen, the endogenous aromatase enzyme in the MCF-7 breast cancer cells (ER-positive cells) could stimulate DNA synthesis, and the stimulation was abolished by the administration of aromatase inhibitors. Burak et al. (1997) demonstrated that androgen elicited an estrogen-induced response in MCF-7 cells through aromatase. These results indicate that aromatase, at a level as found in MCF-7 cells, is capable of generating sufficient estrogen to evoke an estrogen-dependent response. We recently confirmed that, in a steroid-deprived condition, a 5-day incubation of $1 \mathrm{nM}$ estradiol increased the proliferation of MCF-7 cells fourfold, with an $\mathrm{ED}_{50}$ at $0.01 \mathrm{nM}$ (Fig. 1a). It was also found that a 5-day incubation of $10 \mathrm{nM}$ testosterone increased the proliferation of MCF-7 cells threefold, with an $\mathrm{ED}_{50}$ at $1 \mathrm{nM}$ (Fig. 1b). Interestingly, the effective concentrations of testosterone are over ranges similar to the $K_{\mathrm{m}}$ values of testosterone for aromatase (Zhou et al. 1993), suggesting that the growth response of MCF-7 cells to testosterone is correlated with the rate of testosteroneestradiol conversion by aromatase. These experiments demonstrate that in situ estrogen biosynthesis in breast cancer cells can play an autocrine role to promote the growth of cancer cells.

In 1991, an aromatase expressing the MCF-7 cell line (expressing aromatase at a level 10 times that of the untransfected MCF-7 cell line (Zhou et al. 1990)) was prepared in our laboratory and was successfully used as a model to demonstrate an androgen-dependent cell growth (Santner et al. 1993). In addition, tumors were grown in



Estradiol (Molar Concentration in Log Scale)



Testosterone (Molar Concentration in Log Scale)

Figure 1 Induction of the proliferation of MCF-7 cells by estradiol (a) and testosterone (b). MCF-7 cells were cultured in 96-well plates in Eagle's minimal essential medium with non-essential amino acids, sodium pyruvate and Earle's salts, in the presence of $5 \%$ charcoal/dextran-treated serum. The experiments were initiated with 5000 cells per well and were incubated with estradiol or testosterone at the indicated concentrations. After incubation at $37^{\circ} \mathrm{C}$ for 96 ( $\square$ ) and $120(-)$ h, cell proliferation was measured by MTT assay (Tada et al. 1986). 
nude mice inoculated with the aromatase-transfected MCF-7 cells together with Matrigel (Yue et al. 1994). The tumor growth was accelerated by injections of androstenedione. Such nude mouse models are being used to examine the growth responses to aromatase inhibitors. In addition, results obtained from studies using a transgenic mouse model in which aromatase is overexpressed in mammary tissues indicate that in situ produced estrogen plays a more important role than circulating estradiol in breast tumor promotion (Tekmal et al. 1996).

The above-described investigations have generated critical results supporting the theory that in situ aromatase can play a role in promoting breast tumor growth. However, the studies do not address important issues as to the interactions between aromatase-expressing and nonaromatase-expressing cell subpopulations that may affect growth rates and drug (e.g., aromatase inhibitor) sensitivity. In addition, it has not yet been shown whether the tumor cell subpopulations that express aromatase manifest a growth advantage within the tumors. Through a 1-year selection of the previously reported aromatase cDNAtransfected MCF-7 cells with G418, followed by clonal purification, we have obtained an MCF-7 cell line (MCF-7aro) that expresses aromatase in a stable manner, as demonstrated by a steady expression of the enzyme during culture in the absence of G418 (Sun et al. 1997). In order to substantiate the findings made by using MCF-7aro, we also prepared an aromatase-expressing T-47D cell line (T-47Daro) and used it in our study. T-47D cells are also ER-positive. T-47Daro was established through a long-term selection with G418, but without the clonal purification. Therefore, it is possible that T-47Daro contains a mixture of cells expressing different levels of aromatase.

In order to test the hypothesis that tumor aromatase can affect breast tumor growth in a paracrine manner, we have carried out three-dimensional cell culture experiments by co-culturing MCF-7 cells with either MCF-7aro or T-47Daro cells. Testosterone $(1 \mathrm{nM})$ increased cell growth to a similar degree for $\mathrm{MCF}-7 / \mathrm{MCF}-7$ aro co-culture ( 0.75 million cells each type) as with MCF-7aro only (two- to threefold). Testosterone also increased the growth of MCF-7 cells, but to a lesser extent than MCF-7aro or MCF-7/MCF-7aro culture (Sun et al. 1997). In addition, the enzyme specific activities remained unchanged for MCF-7/MCF-7aro co-culture samples with and without androgen treatment, indicating that estrogen produced by transfected cells can also stimulate the growth of untransfected cells. The androgen response could be inhibited by an addition of 4- hydroxyandrostenedione (0.01-0.1 mM). For MCF-7/T-47Daro co-culture experiments, a clear induction of cell growth by androgen was observed and the level of the increase was similar to that on T-47Daro only. However, for either culture with T-47Daro only or with MCF-7/T-47Daro co-culture, the aromatase activity was found to increase significantly after testosterone treatment. Because T-47Daro cells were not subjected to a clonal purification, it is believed that the androgen treatment may selectively stimulate the growth of T-47Daro cells expressing high levels of aromatase. These results indicate that estrogen synthesized by the tumor aromatase can stimulate breast tumor growth in both an autocrine and a paracrine manner.

In summary, aromatase is mainly expressed in adipose stromal cells and fibroblasts in normal breast tissue. The estrogens stimulate epithelial cell growth through a paracrine mechanism. However, in breast tumor tissue, aromatase is found to be expressed in both stromal and cancer cells. Therefore, aromatase stimulates breast tumor growth in both an autocrine and a paracrine manner.

\section{Transcriptional regulation of aromatase expression in breast tumors}

A complex mechanism is involved in the control of human aromatase expression. At least eight exons I have been reported. By performing primer-specific RT-PCR analyses, we and investigators from two other laboratories (Zhou et al. 1996b, Bulun et al. 1997, Harada 1997) have found that exons I.3 and PII are the two major exons I present in aromatase mRNAs isolated from breast tumors. These results suggest that promoters I.3 and II are the major promoters directing aromatase expression in breast cancer and surrounding stromal cells and fibroblasts. These findings indicate that there is a switch of the regulatory mechanism of aromatase expression from normal breast tissue to cancerous tissue. It is known that adipose stromal cells and fibroblasts isolated from noncancerous tissue have mainly exon I.4 containing aromatase mRNA (Harada 1993, Mahendroo et al. 1993).

Characterization of the region upstream of exon I.4 revealed the existence of a TATA-less promoter and an upstream GRE and an Sp1 sequence (Zhao et al. 1955). These elements were shown to be required for expression of reporter gene constructs in the presence of serum and glucocorticoids. In addition, a GAS (interferon- $\gamma$ activating sequence) element was also identified near promoter I.4. Studies from Simpson's laboratory (Michael et al. 1995, 1997) have revealed that promoter II is a cAMP-responsive promoter. In addition, forskolin treatment leads to an increase of the exon I.3-containing message in cultured breast adipose stromal cells (Harada 1997). Therefore, aromatase promoter switches from a glucocorticoid-stimulated promoter, I.4, in normal tissue to cAMP-stimulated promoters, I.3 and II, in cancerous tissue. It is further proposed that an increase of the usage 
of promoters I.3 and II in tumor tissue results from an enhanced cAMP production.

\section{Stimulation of tumor aromatase expression by cAMP}

Investigations from Simpson's laboratory (Michael et al. 1995, 1997) suggest that the Ad4BP/SF-1 element and a CRE-like sequence upstream from promoter II are critical for the cAMP induction of promoter II in ovary tissue. The Ad4BP/SF-1 element reported by Michael et al.(1995) is positioned within the silencer (S1) element identified by us (Zhou \& Chen 1998). As published previously from our laboratory (Wang \& Chen 1992, Zhou \& Chen 1998), the silencer downregulates the transcriptional activity of promoters I.3 and II in breast tissue. Promoter I.3 and S1 were characterized functionally in our laboratory (Zhou etal. 1996a, Zhou \& Chen 1998). Recently, we demonstrated that promoter I.3 is a cAMP-responsive promoter by the demonstration of the induction of its activity by cAMP and by the identification of a CRE, CREaro, between $-66 \mathrm{bp}$ and $-59 \mathrm{bp}$ relative to the transcription start site of promoter I.3. CREaro was identified by DNA deletion and mutation analyses, together with chlorampenicol acetyltransferase (CAT) functional analysis (unpublished observations). These results indicate that promoter I.3 is upregulated by a CRE that is different from that identified for promoter II. Recent RT-PCR analysis from our laboratory has revealed that SF-1 is not expressed in breast cancer tissue in contrast to the ovary (unpublished observations). These findings may explain why cells transfected with a CAT reporter construct containing promoter I.3, the Ad4BP/SF-1 element and the CRE-like sequence $(+16 /+24 \mathrm{bp})$ reported by Michael et al. (1997) do not respond to forskolin treatment (unpublished observations from our laboratory). It may be that cAMP activation of promoter II in breast cancer tissue involves the interaction of the CRElike region $(+16 /+24 \mathrm{bp})$ and a different transcriptional factor that is expressed in breast cancer tissue and binds to the site for Ad4BP/SF-1. Our recent yeast one-hybrid screen studies have identified several nuclear receptors that can bind to this regulatory region (discussed further below).

As, after forskolin treatment, the CAT activity in tumor fibroblasts transfected with a plasmid containing both CREaro and the silencer has been found to be similar to that in cells transfected with a plasmid containing only CREaro (unpublished observations), a functional interaction between the CREaro and the silencer elements is suggested. In the presence of cAMP, the positive regulatory CREaro can overcome the negative regulatory action of the silencer on the promoter function of promoter I.3. We feel that this finding is very important because it provides a molecular basis for the mechanism by which aromatase promoter usage is switched from normal to cancerous tissue. Our current hypothesis is that, in normal breast stromal cells, aromatase expression is regulated by promoter I.4 mediated through glucocorticoid, and the action of promoter I. 3 and II is suppressed by the silencer (Fig. 2a). However, in cancer tissue, cAMP production increases and aromatase promoters are switched to cAMPdependent promoters - that is, I.3 and II (Fig. 2b).

Exon I.6, a newly identified exon I that is upstream from exon I.3 (Fig. 2), was recently reported and detected mainly in THP-1 cells (Shozu et al. 1998). Exon I.6 variants containing exon I.3 have also been found. While we cannot rule out the possibility that some exon I.3containing messages detected in breast tumor tissue by RT-PCR are derived from the unspliced I.6-containing messages, several features of exon/promoter I.6 suggest that exon I.3 detected in breast cancer tissue may not be directly related to exon I.6. Unspliced I.6 variants are minor species in comparison with the fully spliced I.6 and have been detected so far in THP-1 cells, JEG-3 cells, and phorbol myristate (PMA)-treated adipose stromal cells (Shozu et al. 1998). Furthermore, cAMP or forskolin does not stimulate promoter I.6 and actually suppresses the induction of promoter I.6 by PMA or dexamethasone. This may be another control mechanism - cAMP stimulates promoter I.3 and suppresses promoter I.6 in breast tumors.

We have identified another negative regulatory region (labeled as TGF $\beta$ in Fig. 2) immediately upstream from CREaro. This region has not yet been fully characterized.

Aminoglutethimide (AG) is an aromatase inhibitor used to treat estrogen-dependent breast cancer. AG is effective in inhibiting aromatase, but it has been found that aromatase activity in tumors of some breast cancer patients was increased after AG treatment (Miller \& O'Neill 1987). These results may explain why some patients failed to respond to therapy after extensive AG treatment. Recently, we found that AG treatment increased aromatase activity in SK-BR-3, JAR, and HepG2 cell lines in a dose- and incubation time-dependent manner. AG induction is believed to occur at the transcriptional level because the aromatase mRNA level increased after AG treatment in SK-BR-3 and HepG2 cells, as demonstrated by RT-PCR analysis. Furthermore, AG treatment did not increase aromatase activity in aromatase cDNA-transfected cell lines (driven by the $\beta$-actin promoter). Our primer-specific RT-PCR analysis revealed that, in SK-BR-3 cells, AG enhanced the action of a promoter that is different from promoter I.1, I.3, or II. Furthermore, because the AG induction was found to be suppressed by SQ 22536, an adenylate cyclase inhibitor, a cAMP-dependent mechanism might be involved. Therefore, $A G$ induces aromatase expression in the breast 
(a)

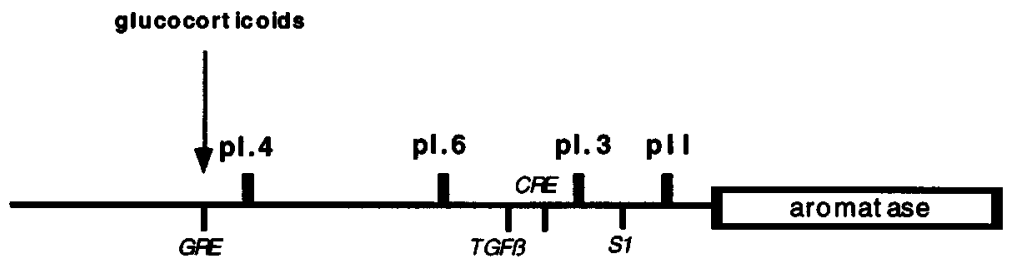

(b)

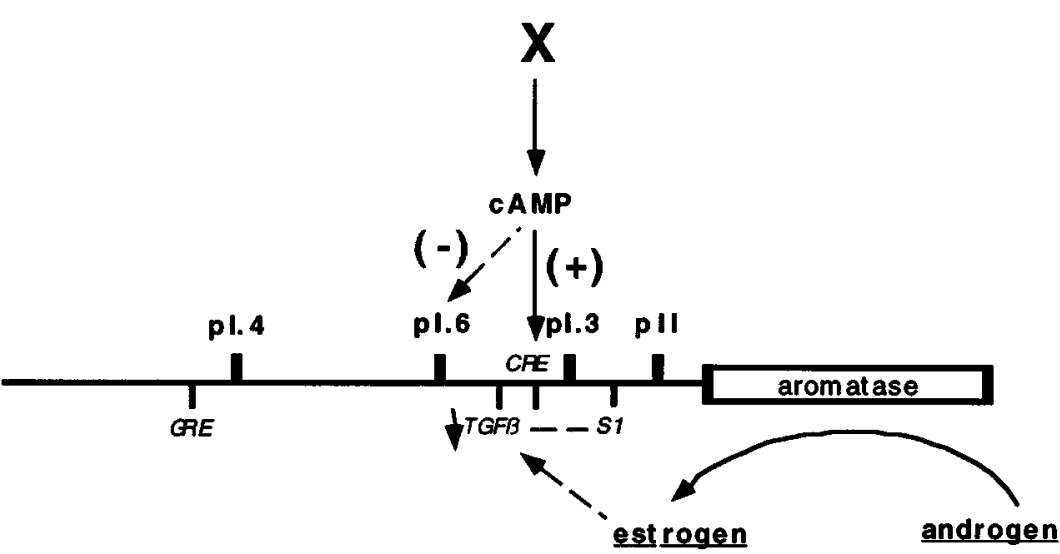

Figure 2 Proposed mechanism of the regulation of aromatase expression in normal breast stromal cells $(a)$ and cancer tissue $(b)$. p, promoter.

cancer tissue through a cAMP-dependent, but not a promoter I.3 (or II)-mediated mechanism.

Results from our own and other laboratories reveal that cAMP plays a critical role in upregulating the expression of aromatase/increasing estrogen biosynthesis in breast cancer tissue. Several factors have been suggested to induce the level of cAMP in breast cancer tissue. For example, Zhao et al. (1997) suggested that prostaglandin $\mathrm{PGE}_{2}$, synthesized in breast cancer cells, induces cAMP response. Furthermore, estrogen is capable of increasing cAMP production in breast cancer cells by stimulating adenylate cyclase (Aronica et al. 1994). These observations suggest a paracrine loop between estrogen production (by aromatase) and cAMP synthesis in breast cancer tissue.

\section{Differential expression of ERR $\alpha-1$ and SF-1 in cells}

Regulation of aromatase expression in breast tumor is also different from that in other tissues by differential usage of transcription factors. This has been found to be the case for the silencer element.
We recently applied the yeast one-hybrid screening method using a breast tissue library to search for proteins binding to the silencer region, and most proteins identified belonged to the nuclear receptor family. Fifty percent of the positive clones encode for ERR $\alpha$-1, and other positive clones include EAR-2, EAR-3 (COUP-TF1), RAR $\gamma$, and p120E4F. As ERR $\alpha-1$ was found to be the major protein interacting with the $\mathrm{S} 1$, we decided to characterize the regulatory action of ERR $\alpha-1$ on promoter I. 3 of the human aromatase gene. Using a reporter plasmid that includes the aromatase genomic fragment containing promoter I. 3 and $\mathrm{S} 1, \mathrm{ERR} \alpha-1$ was found to have a positive regulatory function in breast cancer SK-BR-3 cells (Yang et al. 1998). Gel mobility shift assays have confirmed that ERR $\alpha-1$ binds to $\mathrm{S} 1$ in a dose-dependent manner and DNase I footprinting analysis has revealed that ERR $\alpha-1$ binds to a region within the S1, 5'-AAGGTCAGAAAT-3', between $+96 \mathrm{bp}$ and $+107 \mathrm{bp}$ relative to the transcriptional start site of promoter I.3. Although SF-1 was previously shown to bind to the same site and to function as a CRE in the ovary (Michael et al. 1995, 1997), our yeast onehybrid screening did not find any SF-1 clones. Our RT-PCR analysis was not able to detect SF-1 mRNA in breast cancer tissue and in SK-BR-3 cells. In contrast, our 
RT-PCR analysis identified ERR $\alpha-1$ mRNA in 28 out of 32 breast tumor specimens examined (unpublished observations).

The recent study from our laboratory has indicated that ERR $\alpha-1$ has a positive regulatory effect by interacting with S1. Although it is possible that $\mathrm{S} 1$ functions as a positive regulatory element through an interaction with ERR $\alpha-1, \mathrm{~S} 1$ was previously demonstrated to be a negative regulatory element in several cell lines examined in our laboratory (Wang \& Chen 1992, Zhou \& Chen 1998). Our DNase I footprinting analysis has revealed that ERR $\alpha-1$ binds to the 5'-half region of S1, and at least four proteins were seen in u.v.-crosslinking experiments of S1 using nuclear extract preparations from breast cancer cells and adipose stromal cells (Zhou \& Chen 1998). The negative regulatory action of S1 may result from an interaction of ERR $\alpha-1$ with other nuclear receptors such as EAR-3 (COUP-TF1), which is known to have a negative regulatory function (Klinge et al. 1997), or with co-repressor proteins. As indicated, we have identified EAR-3 during yeast one-hybrid screening, and initial experiments suggest it has a negative regulation of promoter I. 3 in the human aromatase gene (unpublished observations). Gene silencing by EAR-3 is mediated by transcriptional co-repressors, N-CoR and SMRT (Shibata et al. 1997). Co-repressor proteins such as N-CoR and SMRT have a molecular weight of $150 \mathrm{kDa}$, and we have detected proteins with a molecular weight of $150 \mathrm{kDa}$ binding to S1 as demonstrated by u.v.-crosslinking experiments (Zhou $\&$ Chen 1998). The negative action of $S 1$ may result from complex formation among $\mathrm{ERR} \alpha-1$, other nuclear receptors, and co-repressor proteins. It was previously found that an oligonucleotide with the sequence 5'-CCAAGGTCA-3' (a sequence recognized normally by SF-1 or ERR $\alpha-1$ ), at a 50-fold molar excess, was not able to compete with $\mathrm{S} 1$ for nuclear protein binding (Zhou \& Chen 1998). Interestingly, a 50-fold molar excess of S1, but not the oligonucleotide 5'-CCAAGGTCA-3', was found to compete effectively for nuclear protein binding to a radioactive probe with the sequence of 5'-CCAAGGTCA-3'. These results suggest that additional regions of $\mathrm{S} 1$ are involved in the interaction with the silencer-protein complex. This nuclear protein complex has a higher affinity for $\mathrm{S} 1$ than the region recognized by ERR $\alpha-1$ or SF-1. These interactions are being carefully evaluated in our laboratory. It is our current hypothesis that ERR $\alpha-1$ is a transcriptional activator when binding alone to $\mathrm{S} 1$, but its modulating activity can be changed by interacting with co-regulatory proteins.

\section{Perspective}

Suppression of in situ estrogen biosynthesis can be achieved by the prevention of aromatase expression in breast tumors. It is our hope that through an understanding of the regulatory mechanism of aromatase expression in breast cancer tissue, a therapy based on suppressing aromatase expression can be developed. During the past 3 years, we have made significant progress in determining the promoters involved in driving aromatase expression in breast cancer tissue and in determining several important regulatory elements that may affect aromatase expression. We have identified several transcriptional factors that bind to these regulatory elements. We anticipate that we will learn a great deal about the regulatory mechanism of aromatase expression in breast cancer tissue by studying the interaction between transcriptional factors and cisregulatory elements in the genomic region containing promoters I.3 and II. Regulation can also result from the interaction of co-regulatory proteins with transcriptional factors, such as the interaction of co-activator proteins or co-repressor proteins with nuclear receptors.

\section{Acknowledgements}

This research was supported by the National Institutes of Health Grant CA44735. S Chen and D Zhou are members of the City of Hope Breast Cancer Program (CA 65767).

\section{References}

Aronica SM, Kraus WL \& Katzenellenbogen BS 1994 Estrogen action via the cAMP signaling pathway: stimulation of adenylate cyclase and cAMP-regulated gene transcription. Proceedings of the National Academy of Sciences of the USA 91 8517-8521.

Bulun SE, Price TM, Mahendroo MS, Aitken J \& Simpson ER 1993 A link between breast cancer and local estrogen biosynthesis suggested by quantification of breast adipose tissue aromatase cytochrome $\mathrm{P} 450$ transcripts using competitive polymerase chain reaction after reverse transcription. Journal of Clinical Endocrinology and Metabolism 77 1622-1628.

Bulun SE, Noble LS, Takayama K, Michael MD, Agarwal V, Fisher C, Zhao Y, Hinshelwood MM, Ito Y \& Simpson ER 1997 Endocrine disorders associated with inappropriately high aromatase expression. Journal of Steroid Biochemistry and Molecular Biology 61 133-139.

Burak Jr WE, Quinn AL, Farrar WB \& Brueggemeier RW 1997 Androgens influence estrogen-induced responses in human breast carcinoma cells through cytochrome P450 aromatase. Breast Cancer Research and Treatment 44 57-64.

Esteban JM, Warsi Z, Haniu M, Hall PF, Shively JE \& Chen S 1992 Detection of intratumoral aromatase in breast carcinomas, an immunohistochemical study with clinicopathologic correlation. Journal of American Pathology 140 337-343.

Harada N 1993 A unique aromatase (P-450arom) mRNA formed by alternative use of tissue-specific exons 1 in human skin 
fibroblasts. Biochemical and Biophysical Research Communications 189 1001-1007.

Harada N 1997 Aberrant expression of aromatase in breast cancer tissues. Journal of Steroid Biochemistry and Molecular Biology 61 175-184.

James VHT, McNeill JM, Lai LC, Newton CJ, Ghilchik MW \& Reed MJ 1987 Aromatase activity in normal breast and breast tumor tissues: in vivo and in vitro studies. Steroids 50269 279.

Kitawaki J, Fukuoka M, Yamamoto T, Honjo H \& Okada H 1992 Contribution of aromatase to the deoxyribonucleic acid synthesis of MCF-7 human breast cancer cells and its suppression by aromatase inhibitors. Journal of Steroid Biochemistry and Molecular Biology 42 267-277.

Klinge CM, Silver BF, Driscoll MD, Sathya G, Bambara RA \& Hilf R 1997 Chicken ovalbumin upstream promotertranscription factor interacts with estrogen receptor, binds to estrogen response elements and half-sites, and inhibits estrogen-induced gene expression. Journal of Biological Chemistry 272 31465-31474.

Lu Q, Nakamura J, Savinov A, Yue W, Weisz J, Dabbs DJ, Wolz G \& Brodie A 1996 Expression of aromatase protein and messenger ribonucleic acid in tumor epithelial cells and evidence of functional significance of locally produced estrogen in human breast cancers. Endocrinology 1373061 3077.

Mahendroo MS, Mendelson CR \& Simpson ER 1993 Tissuespecific and hormonally controlled alternative promoters regulate aromatase cytochrome $\mathrm{P} 450$ gene expression in human adipose tissue. Journal of Biological Chemistry $\mathbf{2 6 8}$ 19463-19470.

Michael MD, Kilgore MW, Morohashi KI \& Simpson ER 1995 Ad4BP/SF-1 regulates cyclic AMP-induced transcription from the proximal promoter (PII) of the human aromatase P450 (CYP 19) gene in the ovary. Journal of Biological Chemistry 270 13561-13566.

Michael MD, Michael LF \& Simpson ER 1997 A CRE-like sequence that binds CREB amd contributes to cAMPdependent regulation of the proximal promoter of the human aromatase P450 (CYP 19) gene. Molecular and Cellular Endocrinology 134 147-156.

Miller WR \& O'Neill J 1987 The importance of local synthesis of estrogen within the breast. Steroids $\mathbf{5 0} 537-548$.

Miller WR, Mullen P, Sourdaine P, Watson C, Dixon JM \& Telford J 1997 Regulation of aromatase activity within the breast. Journal of Steroid Biochemistry and Molecular Biology 61 193-202.

Pasqualini JR, Chetrite G, Blacker C, Feinstein MC, Delalonde L, Talbi M \& Maloche C 1996 Concentrations of estrone, estradiol, and estrone sulfate and evaluation of sulfatase and aromatase activities in pre- and postmenopausal breast cancer patients. Journal of Clinical Endocrinology and Metabolism 81 1460-1464.

Santen RJ, Martel J, Hoagland M, Naftolin F, Roa L, Harada N, Hafer L, Zaino R \& Santner SJ 1994 Stromal spindle cells contain aromatase in human breast tumors. Journal of Clinical Endocrinology and Metabolism 79 627-632.
Santner SJ, Chen S, Zhou D, Korsunsky Z, Martel J \& Santen RJ 1993 Effect of androstenedione on growth of untransfected and aromatase-transfected MCF-7 cells in culture. Journal of Steroid Biochemistry and Molecular Biology 44 611-616.

Sasano H, Nagura H, Harada N, Goukon Y \& Kimura M 1994 Immunolocalization of aromatase and other steroidogenic enzymes in human breast disorders. Human Pathology 25 530-535.

Shibata H, Nawaz Z, Tsai SY, O’Malley BW \& Tsai MJ 1997 Gene silencing by chicken ovalbumin upstream promotertranscription factor 1 (COUP-TF1) is mediated by transcriptional corepressors, nuclear receptor-corepressor $(\mathrm{N}-\mathrm{CoR})$ and silencing mediator for retinoic acid receptor and thyroid hormone receptor (SMRT). Molecular Endocrinology 11 714-724.

Shozu M, Zhao Y, Bulun SE \& Simpson ER 1998 Multiple splicing events involved in regulation of human aromatase expression by a novel promoter, I.6. Endocrinology 139 16101617.

Sun XZ, Zhou D \& Chen S 1997 Autocrine and paracrine actions of breast tumor aromatase. A three-dimensional cell culture study involving aromatase transfected MCF-7 and T-47D cells. Journal of Steroid Biochemistry and Molecular Biology 63 29-36.

Tada H, Shiho O, Kuroshima KI, Koyama M \& Tsukamoto K 1986 An improved colorimetric assay for interleukin 2. Journal of Immunological Methods 93 157-165.

Tekmal RR, Ramachandra N, Gubba S, Durgam VR, Mantione J, Toda K, Shizuta Y\& Dillehay DL 1996 Overexpression of int-5/aromatase in mammary glands of transgenic mice results in the induction of hyperplasia and nuclear abnormalities. Cancer Research 56 3180-3185.

Wang J \& Chen S 1992 Identification of a promoter and a silencer at the 3'-end of the first intron of the human aromatase gene. Molecular Endocrinology 6 1479-1488.

Yang C, Zhou D \& Chen S 1998 Modulation of aromatase expression in breast tissue by ERR $\alpha-1$ orphan receptor. Cancer Research $\mathbf{5 8}$ 5695-5700.

Yue W, Zhou, Chen S \& Brodie A 1994 A new nude mouse model for postmenopausal breast cancer using MCF-7 cells transfected with the human aromatase gene. Cancer Research 54 5092-5095.

Zhao Y, Mendelson CR \& Simpson ER 1955 Characterization of the sequences of the human CYP19 (aromatase) gene that mediate regulation by glucocorticoids in adipose stromal cells and fetal hepatocytes. Molecular Endocrinology 9 340-349.

Zhao Y, Agarwal VR, Mendelson CR \& Simpson ER 1997 Transcriptional regulation of CYP19 gene (aromatase) expression in adipose stromal cells in primary culture. Journal of Steroid Biochemistry and Molecular Biology 61 203-210.

Zhou D \& Chen S 1998 Characterization of a silencer element in the human aromatase gene. Archives of Biochemistry and Biophysics 353 213-220.

Zhou D, Pompon D \& Chen S 1990 Stable expression of human aromatase cDNA in mammalian cells - a useful system for aromatase inhibitor screening. Cancer Research 50 69496954. 
Zhou D, Wang J, Chen E, Murai J, Siiteri PK \& Chen S 1993 Aromatase gene is amplified in MCF-7 human breast cancer cells. Journal of Steroid Biochemistry and Molecular Biology 46 147-153.

Zhou D, Clarke P, Wang J \& Chen S 1996 a Identification of a promoter that controls aromatase expression in human breast cancer and adipose stromal cells. Journal of Biological Chemistry 271 15194-15202.
Zhou C, Zhou D, Esteban J, Murai J, Siiteri PK, Wilczynski S \& Chen S 1996b Aromatase gene expression and its exon I usage in human breast tumors. Detection of aromatase messenger RNA by reverse transcriptionpolymerase chain reaction (RT-PCR). Journal of Steroid Biochemistry and Molecular Biology 59 163-171. 